\title{
Transurethral resection of the prostate provides more favorable clinical outcomes compared with conservative medical treatment in patients with urinary retention caused by benign prostatic obstruction
}

Yu-Hsiang Lin ${ }^{1,2,4 \dagger}$, Chen-Pang Hou ${ }^{1,2+}$, Tien-Hsing Chen ${ }^{2,5}$, Horng-Heng Juang ${ }^{3}$, Phei-Lang Chang ${ }^{1,2}$, Pei-Shan Yang ${ }^{1,2}$, Chien-lun Chen ${ }^{1,2}$ and Ke-Hung Tsui ${ }^{1,2^{*}}$

\begin{abstract}
Background: To evaluate the long-term surgical outcomes of patients with urinary retention (UR) caused by a benign prostatic obstruction (BPO) who underwent transurethral resection of the prostate (TURP), and compare their outcomes with those of patients who received medication without surgical intervention.

Methods: This retrospective cohort study analyzed claims data collected during the period of 1997-2012 from Taiwan's National Health Insurance Research Database. We examined geriatric adverse events among patients who had received a diagnosis of symptomatic benign prostatic hyperplasia and whom experienced UR, and compared those who received TURP and medication only. Primary outcomes included urinary tract infection (UTI), UR, inguinal hernia, hemorrhoids, stroke, acute myocardial infarction, and bony fracture. We excluded patients who had concomitant prostate cancer, bladder cancer, or a long-term urinary catheter indwelling, as well as those who did not receive a-blocker medication regularly. Those aged $<50$ or $>90$ years were also excluded. The enrolled patients were categorized into TURP $(n=1218)$ and medication only $(n=795)$ groups. After 1:1 propensity score matching, we recorded and compared patients' characteristics, postoperative clinical outcomes, and geriatric adverse events.

Results: The TURP cohort had a lower incidence of UTI and UR during the postoperative follow-up period from 2 months to 3 years than did the medication only group ( $20.7 \%$ vs. $28.9 \%$ and $12.5 \%$ vs. $27.6 \%$, respectively, $p<0.001$ ). The life-long bone fracture incidence was also lower in the TURP cohort (7.9\% vs. 9.2\%, $p=0.048$ ). The incidence of other outcomes during the postoperative follow-up period did not differ between the two groups.
\end{abstract}

Conclusions: Compared with conservative treatment, TURP provides more favorable clinical outcomes in patients with UR caused by BPO. Patients who underwent TURP had a lower risk of UTI, repeat UR episodes, and emergent bony fracture. Thus, early surgical intervention should be considered for such patients.

Keywords: Alfa-blocker, Benign prostate hyperplasia, Urine retention, Outcome, Prostatectomy

\footnotetext{
* Correspondence: khtsui@yahoo.com

${ }^{\dagger}$ Equal contributors

'Department of Urology, Chang Gung Memorial Hospital-Linkou, 5 Fu-Shing

Street, Kweishan, Taoyuan 333, Taiwan, Republic of China

${ }^{2}$ School of Medicine, Chang Gung University, 259 Wen-Hwa 1 st Road,

Kweishan, Taoyuan, Taiwan, Republic of China

Full list of author information is available at the end of the article
} 


\section{Background}

Benign prostatic hyperplasia (BPH) affects approximately 210 million men globally and is a major cause of lower urinary tract symptoms (LUTSs) in aging men [1]. LUTSs negatively affect patients' quality of life and cost the US healthcare system more than $\$ 4$ billion each year [2]. One study estimated that $50 \%$ and $75 \%$ of men have histological evidence of $\mathrm{BPH}$ by the age of 50 and 80 years, respectively, with approximately $50 \%$ of them having clinically significant symptoms [3]. The sequelae of BPH include a decreased urinary flow and advancing voiding and storage symptoms; these may eventually result in acute or chronic urinary retention (UR) [4]. Although men with acute UR caused by BPH have an increased chance of returning to normal voiding if treatment with $\alpha-1$ blockers is started at the time of catheter insertion [5], $24 \%-42 \%$ of patients elect to receive surgical intervention instead $[6,7]$. According to the updated guidelines, surgical intervention is an appropriate treatment for patients with moderate-to-severe LUTSs and for patients who have developed acute UR or other BPH-related complications [8]. Surgical treatment is often effective and prevents the need for indwelling or intermittent catheterization in the future $[9,10]$. However, in a previous study, 79\% of patients received $\alpha 1$ blockers before catheter removal, and most of them could void successfully without requiring an indwelling catheter [11]. To the best of our knowledge, very few studies have compared long-term treatment outcomes between transurethral resection of the prostate (TURP) and medical treatment in patients with BPO who experience UR. Therefore, using data from the National Health Insurance Research Database (NHIRD) of Taiwan, we conducted a nationwide observational cohort study to investigate the surgical outcomes of such patients and compare the long-term treatment outcomes between patients who received medication only and those who underwent surgical intervention.

\section{Methods}

\section{Data source}

We used data from the Longitudinal Health Insurance Database 2000 (LHID2000) in this study. This database contains the claims data of beneficiaries enrolled in the National Health Insurance (NHI) program of Taiwan; to date, more than 4000 research articles have been published using the NHIRD [12]. The LHID2000 includes the claims data of 1000,000 individuals randomly sampled from the entire population enrolled in the NHI program (a total of 23.75 million people) in 2000. The demographic characteristics (i.e., age and sex) between the populations derived from the NHIRD and LHID2000 are not significantly different.

\section{Study design}

We identified patients who had received a diagnosis of $\mathrm{BPH}$ (International Classification of Diseases, Ninth Revision, Clinical Modification [ICD-9-CM] code 600.xx) and had visited the emergency department or an outpatient clinic presenting with UR between January 1, 1997, and December 31,2012 . All identified patients had received $\alpha$-blockers for at least 6 months before the UR episode. UR is defined as indwelling Foley catheterization (Taiwan NHI code 47014C) or intermittent catheterization (Taiwan NHI code 47013C). If patients underwent TURP after BPH with an UR episode, the index date was defined as the discharge date after TURP; otherwise, the index date was defined as the date of the UR episode. Patients who met the following criteria were excluded: (1) age $<50$ years or $>90$ years, (2) a diagnosis of prostate cancer (ICD-9-CM code 185.xx) or bladder cancer (ICD-9-CM code 188.xx) before the index date, (3) regularly receiving $\alpha$-blockers for more than 6 months before the index date, and (4) long-term use of an indwelling urinary catheter. Finally, 2013 patients were included, of which 1218 underwent TURP for UR and 795 received medication without surgical intervention. After 1:1 propensity score matching, we subgrouped the patients into two cohorts: the TURP cohort $(n=736)$ and the medication only cohort $(n=736)$. Finally, patients' characteristics, postoperative clinical outcomes, and geriatric adverse events were recorded and compared. The flow chart for patient enrolment is illustrated in Fig. 1.

\section{Comorbidity detection}

The following comorbidities were included in this study: diabetes mellitus (ICD-9-CM code 250.xx), hypertension (ICD-9-CM codes 401.xx-405.xx), dyslipidemia (ICD-9CM code 272.4), chronic obstructive pulmonary disease (ICD-9-CM codes 491.xx, 492.xx, and 496.xx), Parkinson disease (ICD-9-CM code 332.xx), chronic renal disease or renal failure (ICD-9-CM codes 584.xx and 585.xx), ischemic heart disease (ICD-9-CM codes 410.xx-414.xx), stroke (ICD-9-CM codes 430.xx-437.xx), and heart failure (ICD-9-CM 428.xx). The presence of a comorbidity was ascertained when there was at least one claim of inpatient admission or two claims of outpatient visits 1 year prior to the index date.

\section{Outcome detection}

The outcomes compared in this study were urinary tract infection (UTI) and UR. UTI was defined as hospitalization or an emergency department or outpatient visit with a UTI-related diagnosis (ICD-9-CM codes 599.0x and $595.0 \times$ ) and antibiotic prescription. UR was defined as the occurrence of either an intermittent catheterization or indwelling Foley catheterization. Other geriatric adverse events that were compared included inguinal hernia (Taiwan NHI codes 75606B, 75607C, 75613C, 75614C, and 


\section{Flow chart}

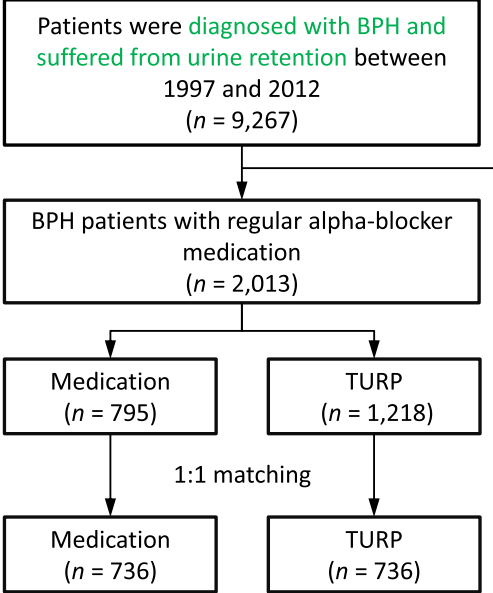

Exclude:

434 Age $<50$ or $>90$ y/o

115 History of prostate cancer

64 History of bladder cance

6,547 Non-regular alpha-blocker users

94 Long-term indwelling catheter

Fig. 1 Flowchart for patient inclusion in this study

75610B), hemorrhoids (Taiwan NHI codes 74406C, 74407C, 74410C, 74411C, 74412C, and 74417C), stroke (ICD-9-CM codes 430.xx-437.xx), and acute myocardial infarction (ICD-9-CM code 410.xx). We also compared the incidence of emergent bone fracture, which was defined as visiting the emergency department or hospitalization with a principal diagnosis of skeletal fracture (ICD-9-CM code 805.xx-829.xx), between the two cohorts, as well as the incidence of urological malignancies after the index date, including prostate cancer (ICD-9-CM code 185.xx) and bladder cancer (ICD-9-CM code 188.9×). The malignancyrelated diagnosis was verified as the possession of a catastrophic illness certificate (CIC) card.

\section{Statistical analyses}

To reduce the possible selection bias and rule out confounding factors, we matched each patient in the TURP group with a counterpart in the medication only group through propensity scoring [13]. The distribution of demographic and clinical characteristics in the TURP and medication only groups was compared using a chi-square test (for the categorical variables) and an independent sample $t$ test (for the continuous variables). The risk of geriatric or urological malignancy-related adverse events during followup was also compared between the study groups by using a Cox proportional hazard model. Additionally, the cumulative survival of bone fracture in the two groups was estimated using the Kaplan-Meier method. Propensity score matching and all statistical analyses were performed using SAS software, version 9.4 (SAS Institute, Cary, NC, USA).

\section{Results}

\section{Study population}

After 1:1 propensity score matching, we grouped the patients into two cohorts: the TURP cohort $(n=736)$ and medication only cohort $(n=736)$ (Table 1$)$. The mean age and follow-up duration of the patients in the TURP and medication only cohorts were 74.2 years (standard deviation $[\mathrm{SD}]=7.9$ years) and 4.2 years $(\mathrm{SD}=3.4$ years $)$, respectively. The mean age and prevalence of comorbidities did not differ significantly between the two cohorts, and the Charlson comorbidity index [14] of the two cohorts was comparable.

\section{Postoperative adverse events}

Geriatric adverse events were compared between the two cohorts to evaluate their association with postoperative adverse events during the 3-year follow-up period (Table 2). Overall, the TURP group had a lower risk of UTI (hazard ratio [HR], 0.62; 95\% confidence interval $[\mathrm{CI}], 0.50-0.76)$ and UR requiring catheterization (HR, 0.35 ; $95 \%$ CI, $0.27-0.45$ ) during the 2 months to 3 years postoperative period. The TURP group also had a lower risk of bone fracture during the postoperative 3-year follow-up period ( $\mathrm{HR}, 0.58$; 95\% CI, 0.37-0.90).

Table 3 presents a comparison of life-long geriatric adverse events between the two groups. Although the life-long incidence of inguinal hernia and hemorrhoids was comparable, the TURP group had a lower risk of life-long bone fracture (HR, 0.70; 95\% CI, 0.49-0.996). Fig. 2 shows the cumulative survival of bone fracture in the two groups, which indicates that the TURP group had a lower risk of bone fracture than did the medication only group.

\section{Urological malignancy-related adverse events}

Urological malignancy-related adverse events were compared between the two groups at the end of the followup period (Table 4). After the index date, prostate cancer was detected in 18 patients $(2.4 \%)$ in the TURP cohort 
Table 1 Patient characteristics after propensity score matching

\begin{tabular}{|c|c|c|c|}
\hline Variable & $\begin{array}{l}\text { TURP group } \\
(n=736) n(\%)\end{array}$ & $\begin{array}{l}\text { Medication group } \\
(n=736) n(\%)\end{array}$ & $P$ value \\
\hline Age (years) & $74.1 \pm 7.5$ & $74.3 \pm 8.4$ & 0.528 \\
\hline \multicolumn{4}{|l|}{ Comorbidity } \\
\hline Diabetes mellitus & $234(31.8)$ & $221(30.0)$ & 0.463 \\
\hline Hypertension & $467(63.5)$ & $455(61.8)$ & 0.518 \\
\hline Hyperlipidemia & $111(15.1)$ & $105(14.3)$ & 0.659 \\
\hline $\begin{array}{l}\text { Chronic obstructive } \\
\text { pulmonary disease }\end{array}$ & $155(21.1)$ & $166(22.6)$ & 0.487 \\
\hline Parkinsonism & $34(4.6)$ & $40(5.4)$ & 0.474 \\
\hline Chronic kidney disease & $114(15.5)$ & $116(15.8)$ & 0.886 \\
\hline Ischemic heart disease & $196(26.6)$ & $179(24.3)$ & 0.309 \\
\hline Stroke & $132(17.9)$ & 137 (18.6) & 0.736 \\
\hline Heart failure & $60(8.2)$ & $63(8.6)$ & 0.778 \\
\hline $\begin{array}{l}\text { Charlson Comorbidity } \\
\text { Index }\end{array}$ & $1.3 \pm 1.2$ & $1.2 \pm 0.9$ & 0.425 \\
\hline
\end{tabular}

Abbreviations: TURP transurethral resection of the prostate

and in 19 patients $(2.6 \%)$ in the medication only cohort during the follow-up period (HR, 0.79; 95\% CI, 0.41-1.50). Furthermore, bladder urothelial carcinoma was detected in 5 patients $(0.7 \%)$ in the TURP cohort and in 10 patients $(1.4 \%)$ in the medication only cohort during the follow-up period (HR, 0.41; 95\% CI, 0.14-1.19). These findings suggest that TURP cannot reduce the incidence of prostate and bladder cancer in patients with BPO who experienced an UR episode.

\section{Discussion}

All clinicians should particularly focus on BPH and BPO, because $50 \%$ of men develop pathological $\mathrm{BPH}$ at the age of 51-60 years [15]. In the United States, the estimated risk of a 50-year-old man with BPH undergoing therapeutic intervention (surgical or medical treatment) in his lifetime is approximately 40\% [16]. A 3-year, multicenter, randomized controlled trial investigated patients with moderate $\mathrm{BPH}$ symptoms who were treated through either watchful waiting or TURP. In this trial, $24 \%$ and $2.9 \%$ of men in the watchful waiting arm crossed over to receive surgical intervention developed UR, respectively [17]. UR, one of the common complications of BPH and $\mathrm{BPO}$, is a distressing urological emergency that seriously affects patients' health and quality of life. Among men aged 70-79 years with $\mathrm{BPH}$ and moderate-to-severe LUTSs (IPSS score $>7$ ), the incidence of UR was determined to be approximately 34.7 per 1000 person-years [18]. Another large-scale study that evaluated an ethnically diverse group of males in the United States reported that the observed incidence of $\mathrm{BPH}$-associated UR increased substantially between 2007 and 2010 [19].

In men with $\mathrm{BPH}$, risk factors for UR include advanced age, severe LUTSs, increased prostate volume, decreased urinary flow rate, and prostate-specific antigen level $>2.5$ [20]. Three factors predominate the pathophysiological mechanisms of UR: outflow obstruction, neurological impairment, and an inefficient detrusor muscle [21], among which outflow obstruction is the most common cause [22]. Another urodynamic study on UR reported that outflow obstruction may develop secondary to the interruption of sensory or motor nerve supply to the detrusor muscle, incomplete relaxation of the urinary sphincter mechanism, or inefficient contraction of the bladder detrusor muscle [23].

Once acute UR occurs, the initial management includes immediate decompression of the urinary bladder through urethral Foley catheterization or indwelling suprapubic cystostomy if urethral catheterization is not possible [24-26]. Although UR is one of the absolute indicators for surgical treatment in patients with $\mathrm{BPH} /$ BPO [27], TURP is not the first choice of treatment in daily practice because of its potential risks and complications. Instead, $\alpha$-blockers, which improve BPO in men with LUTSs, are regarded as the first-line treatment for BPO [28]. $\alpha$-Blockers can result in a successful trial without catheter (TWOC) in patients with acute UR. Some urologists offer a trial of voiding to patients with acute UR, and one study reported that patients voided successfully by 12 weeks after TWOC without surgical treatment [29]. Another study reported that $48 \%$ of

Table 2 Geriatric adverse events during the 3-year follow-up period

\begin{tabular}{|c|c|c|c|c|}
\hline \multirow[t]{2}{*}{ Variable } & \multirow[t]{2}{*}{ TURP $(n=736) \mathrm{n}(\%)$} & \multirow[t]{2}{*}{ Medication $(n=736) \mathrm{n}(\%)$} & \multicolumn{2}{|c|}{ TURP vs. Medication } \\
\hline & & & HR $(95 \% \mathrm{Cl})$ & $P$ value \\
\hline UTI (post-op 2 months - 3 years) & $152(20.7)$ & $213(28.9)$ & $0.62(0.50,0.76)$ & $<0.001$ \\
\hline UR (post-op 2 months - 3 years) & $92(12.5)$ & $203(27.6)$ & $0.35(0.27,0.45)$ & $<0.001$ \\
\hline Inguinal hernia & $18(2.4)$ & $17(2.3)$ & $0.96(0.50,1.87)$ & 0.914 \\
\hline Hemorrhoids & $9(1.2)$ & $9(1.2)$ & $0.92(0.37,2.33)$ & 0.867 \\
\hline Stroke & $28(3.8)$ & $25(3.4)$ & $1.01(0.59,1.73)$ & 0.985 \\
\hline AMI & $10(1.4)$ & $11(1.5)$ & $0.77(0.33,1.82)$ & 0.550 \\
\hline Fracture & $31(4.2)$ & $48(6.5)$ & $0.58(0.37,0.90)$ & 0.016 \\
\hline
\end{tabular}

Abbreviations: TURP transurethral resection of the prostate, $H R$ hazard ratio, $C l$ confidence interval, $A M I$ acute myocardial infarction 
Table 3 Comparison of life-long geriatric adverse events

\begin{tabular}{lllll}
\hline Variable & TURP $(n=736) n(\%)$ & $\begin{array}{l}\text { Medication } \\
(n=736) n(\%)\end{array}$ & & \multicolumn{2}{l}{ TURP vs. Medication } \\
\cline { 4 - 6 } & & $32(4.3)$ & $0.70(0.42,1.17)$ & 0.169 \\
\hline $\begin{array}{l}\text { Inguinal } \\
\text { hernia }\end{array}$ & $27(3.7)$ & $15(2.0)$ & $0.74(0.35,1.55)$ & 0.425 \\
Hemorrhoids & $13(1.8)$ & $42(5.7)$ & $1.03(0.68,1.54)$ & 0.906 \\
Stroke & $51(6.9)$ & $15(2.0)$ & $1.13(0.58,2.20)$ & 0.719 \\
AMl & $21(2.9)$ & $68(9.2)$ & $0.70(0.49,0.996)$ & 0.048 \\
Fracture & $58(7.9)$ & &
\end{tabular}

Abbreviations: TURP transurethral resection of the prostate, $H R$ hazard ratio, $\mathrm{Cl}$ confidence interval, $A M I$ acute myocardial infarction

patients with acute UR had a successful TWOC when they were administered an $\alpha$-blocker (Tamsulosin), whereas only $26 \%$ of patients had a successful trial when no drug was administered [30]. Elsewhere, researchers indicated that after initial catheterization, $72.8 \%$ of men had a successful TWOC after a median of 3 days of catheterization, of which $79 \%$ had received an $\alpha 1-$ blocker (Alfuzosin) before catheter removal [11].

Although treatment with $\alpha$-blockers without surgical intervention can result in a successful TWOC in patients with acute UR, studies have yet to demonstrate the long-term clinical outcomes of these patients. Therefore, the present study compared long-term clinical outcomes between patients who had received TURP and those who had received medication only by using data from Taiwan's NHIRD. Before comparing the clinical outcomes of the two cohorts, we performed 1:1 propensity score matching [13] to ensure that the characteristics of the two groups were similar and more objective data could be obtained. Therefore, the distribution of age, incidence of preoperative comorbidities, and Charlson comorbidity index did not differ significantly between the two groups (Table 1).

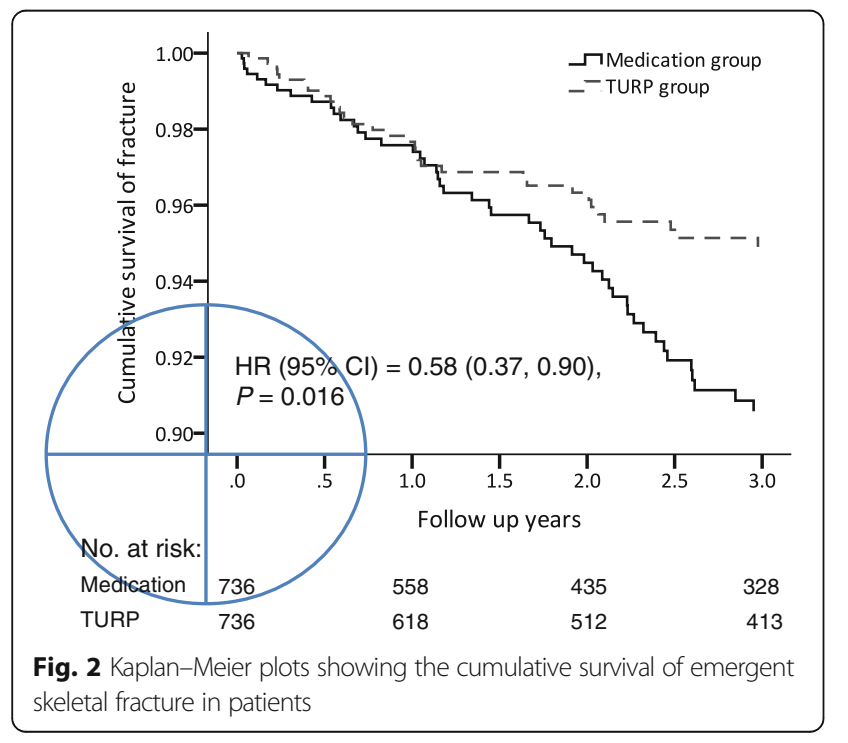

Table 4 Urological malignancy-related adverse events at the end of follow-up

\begin{tabular}{lllll}
\hline Variable & $\begin{array}{l}\text { TURP } \\
(n=736)\end{array}$ & $\begin{array}{l}\text { Medication } \\
(n=736)\end{array}$ & \multicolumn{2}{l}{ TURP vs. Medication } \\
\cline { 4 - 5 } & $18(2.4)$ & $19(2.6)$ & $0.79(0.41,1.50)$ & 0.463 \\
\hline $\begin{array}{l}\text { Prostate } \\
\text { adenocarcinoma }\end{array}$ & & $10(1.4)$ & $0.41(0.14,1.19)$ & 0.102 \\
$\begin{array}{l}\text { Bladder urothelial } \\
\text { carcinoma }\end{array}$ & $5(0.7)$ & & & $P$ value \\
\hline
\end{tabular}

Abbreviations: TURP transurethral resection of the prostate, $H R$ hazard ratio, $\mathrm{Cl}$ confidence interval

Moderate-to-severe LUTSs considerably affect all parameters of quality of life for aging men [31], and appropriate management is warranted. TURP is a safe and effective surgical procedure for men with $\mathrm{BPH}$ and moderate-to severe LUTSs. TURP can even achieve favorable outcomes in stroke and DM patients with symptomatic benign prostate hyperplasia [32, 33]. All of the patients included in our study had received $\alpha$ blockers for at least 6 months before experiencing an UR episode. Our results showed that clinical outcomes were more favorable in the TURP group compared with the medication only group, because the TURP group had a lower risk of UTI and UR. Furthermore, the TURP group had a lower incidence of future emergent skeletal fracture during both postoperative 3-year follow-up and life-long observation periods.

Nocturia is not only the leading cause of sleep fragmentation in older adults [34] but also a crucial risk factor for falls among men older than 65 years [35]. A study based on the Japanese National Health Insurance system reported that elderly individuals with nocturia had a higher risk of fracture and death than did those without nocturia [36]. Another study also reported an association of nocturia with a higher risk of comorbidities, such as bone fracture, diabetes, and coronary disease, and thus a higher risk of mortality among elderly individuals [37]. In patients who had experienced acute UR, TURP probably resulted in more favorable treatment outcomes compared with medication alone in terms of LUTSs, including nocturia. LUTSs were relieved once a patient received TURP. TURP appears to reduce the urge and prompt sensation to void and the number of times a patient gets up to visit the toilet at night, thus preventing them from the risk of falls. On the other hand, orthostatic hypotension is an independent risk factor for recurrent falls among the elderly [38]. After receiving TURP, the chance of a patient taking alpha-blockers would probably decrease, thereby reducing the possibility of fall caused by postural hypotension, which is the possible side effect of alphablockers.

In this study, we also investigated whether the medication only group had a higher future incidence of urological 
malignancy. Given that high post-voiding residual urine, repeat UTI, chronic bladder inflammation, and chronic UR all increase the urothelial exposure to carcinogens [39], we hypothesized that the medication only group would have a higher future incidence of bladder urothelial carcinoma. However, our data revealed that the incidence of both bladder urothelial carcinoma and prostate adenocarcinoma was identical in the two cohorts. This may be because the number of patients with malignancy in this study was too small to observe any statistical difference.

This study has some limitations that were inherited from the data structure of the NHIRD. First, this database does not provide detailed personal information, such as laboratory parameters, alcohol consumption, cigarette use, and exercise, which are confounding variables that influence LUTSs and bladder urothelial carcinoma. Some important reports like pre-operative prostate volumes and the urodynamic studies of the patients were not obtained in this study, either. Second, we used strict dichotomy to divide our study population into two groups: the TURP and medication only groups. Thus, we could not assess whether the time length from acute UR to surgery or whether the number of UR episodes affected treatment outcomes. Third, the use of prostatic vaporization (or ablation by laser), which is not reimbursed by the Taiwan NHI, has only become increasingly common in the last decade [40]. Thus, patients receiving prostate laser treatment were not included in this database. However, despite these limitations, this is the first study to compare the long-term treatment outcomes of TURP and medication only for patients who experience acute UR. Thus, we believe this is innovative and valid research.

\section{Conclusions}

Although treatment with $\alpha$-blockers without surgical intervention can result in a successful TWOC in patients with $\mathrm{BPH} / \mathrm{BPO}$ who experience acute UR, TURP provides more favorable long-term clinical outcomes. The patients who received TURP had a lower risk of UTI, repeat UR episodes, and emergent bony fracture in the future than did those who received medication alone. We conclude that early surgical intervention should therefore be considered for such patients.

\section{Abbreviations \\ $\mathrm{BPH}$ : Benign prostate Hypertrophy; BPO: Benign prostatic obstruction; LUTS: Lower urinary tract symptoms; NHI: National Health Insurance; TURP: Transuretrhal resection of prostate; UR: Urine retention}

\section{Acknowledgements}

We would like to thank all the participants, and colleagues at Chang Gung Memorial Hospital who made this study possible.

\section{Funding}

This work was supported by Chang Gung Research Program (Grant numbers: CRPG3A1061/CMRPG3A1062,CMRPG3E0151-3/CMRPF0801-3,from the Chang
Gung Memorial Hospital and Ministry of Science and Technology (NSC104-2314-B-182A-140-MY3) from the Promotion of National SCI-Tec program. The funders had no role in study design, data collection and analysis, decision to publish, or preparation of the manuscript.

\section{Availability of data and materials}

The datasets and/or analysed during the current study are available from the corresponding author on reasonable request.

\section{Authors' contributions}

Study conception and design: KHT, HHJ, YHL, CPH, THC, PLC, PSY, CLC. Acquisition of data: KHT, HHJ. Analysis and interpretation of the data: $Y H L$, $\mathrm{CPH}, \mathrm{THC}$. Drafting the paper: PLY, PLC, CLC. Critical revision: HHJ, KHT, YHL. All authors read the manuscript and approved the submission.

\section{Ethics approval and consent to participate}

We obtained written consent from the participants. The protocol of this project was approved by the Ethics Committee on Chang Gung Memorial Hospital Ethics Board at the CGMH, Taiwan, 104-7627B.

\section{Competing interests}

The authors declare that they have no competing interests.

\section{Publisher's note}

Springer Nature remains neutral with regard to jurisdictional claims in published maps and institutional affiliations.

\section{Author details}

${ }^{1}$ Department of Urology, Chang Gung Memorial Hospital-Linkou, 5 Fu-Shing Street, Kweishan, Taoyuan 333, Taiwan, Republic of China. ${ }^{2}$ School of Medicine, Chang Gung University, 259 Wen-Hwa 1 st Road, Kweishan, Taoyuan, Taiwan, Republic of China. ${ }^{3}$ Department of Anatomy, School of Medicine, Chang Gung University, Kwei-shan, Tao-Yuan, Taiwan, Republic of China. ${ }^{4}$ Graduate Institute of Clinical Medical Sciences, College of Medicine, Chang Gung University, Taoyuan, Taiwan, Republic of China. ${ }^{5}$ Division of Cardiology, Department of Internal Medicine, Chang Gung Memorial Hospital, 222, Maijin Road, Keelung, Taiwan, Republic of China.

Received: 22 October 2017 Accepted: 3 January 2018

Published online: 16 January 2018

\section{References}

1. Verhamme KM, Dieleman JP, Bleumink GS, van der Lei J, Sturkenboom MCJM. Incidence and prevalence of lower urinary tract symptoms suggestive of benign prostatic hyperplasia in primary care - the triumph project. Eur Urol. 2002;42:323-8.

2. Saigal CS, Joyce G. Economic costs of benign prostatic hyperplasia in the private sector. J Urol. 2005;173:1309-13.

3. Guess HA, Arrighi HM, Metter EJ, Fozard JL. Cumulative prevalence of prostatism matches the autopsy prevalence of benign prostatic hyperplasia. Prostate. 1990;17:241-6.

4. Jacobsen SJ, Jacobson DJ, Girman CJ, et al. Natural history of prostatism: risk factors for acute urinary retention. J Urol. 1997;158:481-7.

5. Selius BA, Subedi R. Urinary retention in adults: diagnosis and initial management. Am Fam Physician. 2008;77(5):643-50.

6. Pickard R, Emberton M, Neal DE. The management of men with acute urinary retention. National Prostatectomy Audit Steering Group. Br J Urol. 1998;81:712-20

7. Emberton M, Neal DE, Black N, et al. The National Prostatectomy Audit: the clinical management of patients during hospital admission. Br J Urol. 1995; 75:301-16.

8. Davidian MH. Guidelines for the treatment of benign prostatic hyperplasia. US Pharm. 2016;41:36-40.

9. Ghalayini IF, Al-Ghazo MA, Pickard RS. A prospective randomized trial comparing transurethral prostatic resection and clean intermittent selfcatheterization in men with chronic urinary retention. BJU Int. 2005:96:93-7.

10. Gujral S, Abrams P, Donovan $J$, et al. A prospective randomized trial comparing transurethral resection of the prostate and laser therapy in men with chronic urinary retention: the CLasP study. J Urol. 2000;164:59-64. 
11. Desgrandchamps F, De La Taille A, DOUBLET JD. The management of acute urinary retention in France: a cross-sectional survey in 2618 men with benign prostatic hyperplasia. BJU Int. 2006;97:727-33.

12. Hsiao F, Yang C, Huang Y, Huang W. Using Taiwan's national health insurance research databases for pharmacoepidemiology research. J Food Drug Anal. 2007;15(2):99.

13. Charlson ME, Pompei P, Ales KL, McKenzie CR. A new method of classifying prognostic comorbidity in longitudinal studies: development and validation. J Chron Dis. 1987;40:373-83.

14. Berry SJ, Coffey DS, Walsh PC, Ewing LL. The development of human benign prostatic hyperplasia with age. J Urol. 1984;132:474.

15. Oesterling JE. Benign prostatic hyperplasia: a review of its histogenesis and natural history. Prostate. 1996;29:67-73.

16. Walsh PC. A comparison of transurethral surgery with watchful waiting for moderate symptoms of benign prostatic hyperplasia. J Urol. 1995;154:301.

17. Jacobsen SJ, Jacobson DJ, Girman CJ, Roberts RO, Rhodes T, Guess HA, Lieber MM. Natural history of prostatism: risk factors for acute urinary retention. J Urol. 1997;158:481-7.

18. Groves HK, Chang D, Palazzi K, Cohen S, Parsons JK. The incidence of acute urinary retention secondary to $\mathrm{BPH}$ is increasing among California men. Prostate Cancer Prostatic Dis. 2013:16:260-5.

19. Lieber MM, Rhodes T, Jacobson DJ, McGree ME, Girman CJ, Jacobsen SJ, et al. Natural history of benign prostatic enlargement: long-term longitudinal population-based study of prostate volume doubling times. BJU Int. 2010; 105(2):214-9.

20. Choong S, Emberton M. Acute urinary retention. BJU Int. 2000;85:186.

21. Curtis LA, Dolan TS, Cespedes RD. Acute urinary retention and urinary incontinence. Emerg Med Clin North Am. 2001;19:591.

22. Murray K, Massey A, Feneley RC. Acute urinary retention-a urodynamic assessment. Br J Urol. 1984;56:468.

23. Khan M, Khan AL, Khan AS, Nawaz H. Benign prostatic hyperplasia: mode of presentation and postoperative outcome. J Pak Med Assoc. 2005;55:20-3.

24. Hallett JM, Stewart GD, McNeill SA. The Management of Acute Urinary Retention: treating the curse of the aging male. Curr Bladder Dysfunct Rep. 2013;8:242-9.

25. Fitzpatrick JM, Kirby RS. Management of acute urinary retention. BJU Int. 2006;97:16-20.

26. Oelke M, Bachmann A, Descazeaud A, Emberton M, Gravas S, Michel MC, N'Dow J, Nordling J, Jean J. EAU guidelines on the treatment and follow-up of non-neurogenic male lower urinary tract symptoms including benign prostatic obstruction. Eur Urol. 2013;64:118-40.

27. Fusco F, Palmieri A, Ficarra V, Giannarini G, Novara G, Longo N, Verze P, Creta $M$, Mirone V. a1-blockers improve benign prostatic obstruction in men with lower urinary tract symptoms: a systematic review and meta-analysis of urodynamic studies. Eur Urol. 2016;69:1091-101.

28. Hagiwara K, Koie T, Iwamura H, Imai A, Hatakeyama S, Yoneyama T, Hashimoto Y, Ohyama C. Efficacy and safety of Silodosin and Dutasteride combination therapy in acute urinary retention due to benign prostatic hyperplasia: a single-arm prospective study. Biomed Res Int. 2016;2016: 4975851. https://doi.org/10.1155/2016/4975851. Epub 2016 Apr 18.

29. Lucas MG, Stephenson TP, Nargund V. Tamsulosin in the management of acute urinary retention from benign prostatic hyperplasia. BJU Int. 2005;95(3):354-7.

30. Rosenbaum PR, Donald BR. The central role of the propensity score in observational studies for causal effects. Biometrika. 1983;70:41-55.

31. Perchon LF, Pintarelli VL, Bezerra $E$, Thiel M, Dambros M. Quality of life in elderly men with aging symptoms and lower urinary tract symptoms (LUTS). Neurourol Urodyn. 2011:30:515-9.

32. Hou CP, Lin $\mathrm{YH}$, Chen $\mathrm{TH}$, Chang $\mathrm{PL}$, Juang HH, Chen $\mathrm{CL}$, Tsui $\mathrm{KH}$. Transurethral resection of the prostate achieves favorable outcomes in stroke patients with symptomatic benign prostate hyperplasia. Aging Male. 2017;1-8. https://doi.org/10.1080/13685538.2017.1358260.

33. Lin YH, Hou CP, Chen TH, Juang HH, Chang PL, Yang PS, Tsui KH. Is diabetes mellitus associated with clinical outcomes in aging males treated with transurethral resection of prostate for bladder outlet obstruction: implications from Taiwan Nationwide population-based cohort study. Clin Interv Aging. 2017;12:535.

34. Bliwise DL, Foley DJ, Vitiello MV, Ansari FP, Ancoli-Israel S, Walsh JK. Nocturia and disturbed sleep in the elderly. Sleep Med. 2009;10:540-8.

35. Parsons JK, Mougey J, Lambert L, Wilt TJ, Fink HA, Garzotto M, Marshall LM. Lower urinary tract symptoms increase the risk of falls in older men. BJU Int. 2009;104:63-8.
36. Nakagawa H, Niu K, Hozawa A, Ikeda Y, Kaiho Y, Ohmori-Matsuda K, Tsuji I. Impact of nocturia on bone fracture and mortality in older individuals: a Japanese longitudinal cohort study. J Urol. 2010;184:1413-8.

37. Asplund R. Mortality in the elderly in relation to nocturnal micturition. BJU Int. 1999;84:297-301.

38. Ooi WL, Hossain M, Lipsitz LA. The association between orthostatic hypotension and recurrent falls in nursing home residents. Am J Med. 2000; 2:106-11.

39. Burger M, Catto JW, Dalbagni G, Grossman HB, Herr H, Karakiewicz P, Lotan Y. Epidemiology and risk factors of urothelial bladder cancer. Eur Urol. 2013; 63:234-41.

40. Lee WC, Lin YH, Hou CP, et al. Prostatectomy using different lasers for the treatment of benign prostate hyperplasia in aging males. Clin Interv Aging. 2013;8:1483-8.

\section{Submit your next manuscript to BioMed Central and we will help you at every step:}

- We accept pre-submission inquiries

- Our selector tool helps you to find the most relevant journal

- We provide round the clock customer support

- Convenient online submission

- Thorough peer review

- Inclusion in PubMed and all major indexing services

- Maximum visibility for your research

Submit your manuscript at www.biomedcentral.com/submit
) Biomed Central 